\title{
Atomic-Resolution Study of Grain Boundaries in CdTe Using Scanning Transmission Electron Microscopy
}

Jinglong Guo, ${ }^{1}$ Fatih G. Sen, ${ }^{2}$ Luhua Wang, ${ }^{3}$ Seungjin Nam, ${ }^{3}$ Moon Kim, ${ }^{3}$ Maria K. Y. Chan, ${ }^{2}$ Robert F. Klie, ${ }^{1}$

1. University of Illinois at Chicago, Department of Physics, Chicago, IL.

2. Argonne National Laboratory, Center for Nanoscale Materials, Argonne, IL.

3. University of Texas at Dallas, Department of Materials Science and Engineering, Dallas, TX.

CdTe is one of the most promising photovoltaic materials due to its near optimum band gap and a high absorption coefficient. However, the efficiencies of both champion poly-crystalline CdTe photovoltaic cells as well as production line modules are still significantly below the theoretical Shockley-Queisser limit of $\sim 30 \%$.[1-2] Reduction of non-radiative recombination at grain boundaries is believed to be the key to improving the efficiency of polycrystalline CdTe-based solar cells. [3] Atomistic-level characterization, including scanning transmission electron microscopy (STEM) and first principles density functional theory (DFT) modeling, is crucial in developing a fundamental understanding of how grain boundaries affect the solar cells' efficiency.[4]

In this contribution, we examine grain boundaries in poly-crystalline CdTe solar-cell samples and compare the interfacial atomic and electronic structures with model-system CdTe grain boundaries using ultra-high vacuum bonded bicrystals.[4] Atomic-resolution characterization is carried out in the JEOL ARM200CF aberration-corrected scanning transmission electron microscope (STEM) using high-angle annular dark field (HAADF) and annular bright field (ABF) imaging. The EELS mapping was acquired using a the Gatan Quantm GIF to determine the chemical composition across the grain boundaries. Electronic defect structures are studied using first-principles density functional theory (DFT) calculations. These calculations were performed on structural models based on atomic-resolution STEM images.

Figure 1a) shows a low magnification HAADF STEM image of a poly-crystalline solar cell sample grown by close-space sublimation (CSS) in the superstrate configuration. The image clearly shows large grains on top of the transparent conductive oxide layer. The individual grains also contain a high concentration of extended defects either in the form of twin boundaries or stacking faults. Figure 1b) shows an EELS spectrum image using the $\mathrm{Cl} L$-edge, demonstrating that $\mathrm{Cl}$ segregation along the $\mathrm{CdTe}$ grain boundaries. Figure 1c) shows the calculated density of states (DOS) using DFT for a dislocation core typically found in CdTe grain boundaries. The DOS shows a high intensity of mid-gap states in pristine dislocation core. These mid-gap states are responsible for the high rates of carrier recombination at the grain boundaries. Our DFT calculations shown that the presence of $\mathrm{Cl}$ at the grain boundaries significantly reduces the density of midgap states, thus effectively passivating the CdTe grain boundaries (Figure 1c). Figure 1d) shows our DFT calculation that predict that replacing Te with Se within the dislocation core is even more efficient in reducing mid-gap states. Using CdTe bi-crystals, the effects of various passivants on the grain boundary structures, such as a mixed $45^{\circ}$ twist with $11^{\circ}$ tilt boundary (Figure 1e) will be examined. The structural model for the two grains in the [100] and [110] zone axis orientation is shown superimposed on Figure 1e) and will be used as input for our DFT modeling. [5] 
References:

[1] Green M.A et al, Dunlop, Prog. Photovolt.: Res. Appl. 23 (2014), p. 1.

[2] Shockley, W. et al, Journal of Applied Physics 32 (3) (1961), p. 510.

[3] Paulauskas T. et al, Acta Crystallographica Section A Foundations and Advances 70(6) (2014), p. 524.

[4] Sun, C. et al, Scientific Reports, 6(1) (2016), p. 27009.

[5] This work is supported by the U.S. Department of Energy's Office of Enery Efficiency and Renewable Energy (EERE) under Solar Energy Technology Office (SETO) Award Number DEEE00007545. Use of the Center for Nanoscale Materials, an Office of Science user facility, was supported by the U. S. Department of Energy, Office of Science, Office of Basic Energy Sciences, under Contract No. DE-AC02-06CH11357.
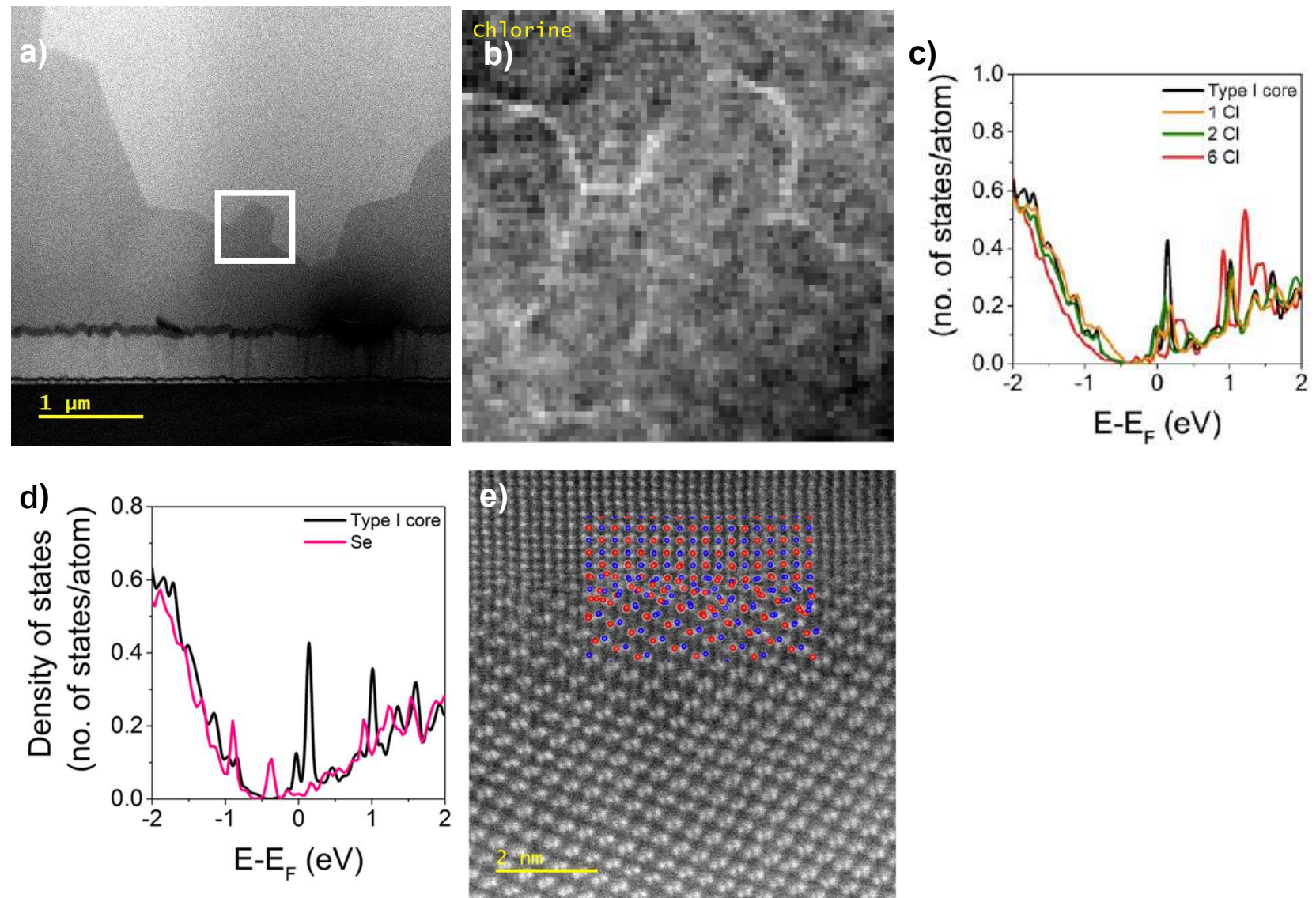

Figure 1. a) HAADF image of poly-crystalline CdTe solar cell device where the glass substrate and transparent conductive oxide front contact are shown on the bottom of the image. Several large grains sizes are clearly visible. b) EELS Mapping of $\mathrm{Cl}$ segregation along the grain boundaries from an area shown in a). c) The effect of $\mathrm{Cl}$ passivation on the density of midgap states at the grain boundary. d) DOS of a dislocation core with and without Se passivation. e) HAADF image of a mixed grain boundary found in a CdTe bi-crystal. d) 